\title{
Multimedia Presentation of Spatial Journalistic Content
}

\author{
Zeinab Torabi ${ }^{\text {a }}$, Jan Wilkening ${ }^{b}$, Juliane Cron ${ }^{\mathrm{a}, *}$ \\ ${ }^{a}$ Technical University of Munich, Chair of Cartography, z.torabi.19@gmail.com, juliane.cron@tum.de \\ ${ }^{b}$ Esri Deutschland GmbH, j.wilkening@esri.de \\ * Corresponding author
}

\begin{abstract}
:
Everything happens somewhere. Thus, location plays an important role in data journalism. As interactive websites have become the standard medium for telling journalistic stories, web mapping and multimedia frameworks are often integrated to tell them in an audio-visually appealing way. Often, these integrated frameworks are developed by GIS or web mapping companies in order to reach new target groups such as journalists.

In this study, we investigated different approaches for integrating web maps and multimedia presentations in a journalistic story. We first defined the most important criteria for a well-suited multimedia presentation of spatial journalistic content. We also identified the most relevant platforms for presenting spatial journalistic content, based on popularity and completeness. Finally, we built a sample story to show how web maps and multimedia elements can be used to represent spatial journalistic content. We built this story with the two most suitable frameworks and evaluated the story with experts and according to our criteria.
\end{abstract}

Keywords: Data Journalism, Multimedia, Storytelling

\section{Introduction}

Presenting journalistic information through online media has become of a particularly great interest with the steady increase of online users. Nowadays, more and more people read news online, for example, while they are in public transportation. Online platforms are capable of updating the latest news within a few minutes and their users are only a few clicks away from them. This medium has also enabled journalists to depict a story with the most powerful combination of multimedia tools, such as photo, audio, video, animated information graphics or interactive maps. Many of these multimedia articles or stories are directly or indirectly linked to location. However, despite this abundant appearance of spatial data in online journalism, there are not many studies done in this area. Even though multimedia and interactivity are common features in online journalism, many stories based on spatial data are not making use of the potential of such a presentation offer.

\section{Criteria for creating integrated multimedia web mapping applications}

In order to conclude the most important criteria for creating integrated multimedia-web mapping applications, literature in areas such as "multimedia in journalism", "spatial journalistic content", "digital narrative storytelling" and "web mapping" has been reviewed.

Jacobson (2012) examines multimedia packages that are produced by nytimes.com ${ }^{1}$ between $2000-2008$. In her study, the multimedia packages are divided into four distinct categories: 'Video', 'Audio', 'Slide Show', and 'Interactive Features'. This article gives an insight into the significance of 'Interactive Features' in the 'Feature' category of news stories. The examples of 'Interactive Features' include interactive infographics, interactive web maps, and interactive timelines. Smiciklas (2012) defines infographics as data visualization that are intended to convey complex information or ideas more quickly and clearly.

Taking the important role of multimodality in journalism into account, the feature of multimedia integration is selected as one of the vital requirements. Moreover, some of the selected elements within the test story, which will be explained later, are inspired by the commonly used multimedia practices within journalistic articles.

Regarding the spatial data in multimedia presentations, there are a few factors to be considered, such as spatial data type capabilities or the possibility of spatial analysis. Furthermore, taking the powerful impact of maps on people's geopolitical perception, it is important to consider a platform which everyone can use (either an expert programmer, a geographic specialist or a completely nonprofessional user) and which has a wide and affordable accessibility.

Churchill and Stege (2006) assert that apart from the geographic consideration of the spatial data, the messages conveyed by journalistic maps are of greater importance. Feigenbaum et al. (2016) argue that while maps are proliferating in the news, there is currently a lack of

\footnotetext{
${ }^{1}$ https://www.nytimes.com
} 
journalistic education on how power inequalities can get embedded and reproduce through these visualizations.

Also, from a user perspective, user-friendliness of the interface is another key factor which needs to be considered. Black and Cartwright (2005) explore the capabilities of web-enabled GIS that used to be limited only to desktop environment. They emphasise on providing a flexible and intuitive interface of GIS tools for non-GIS users, so that everyone can better understand and present spatial data. Another aspect that is essential for an ideal platform is that it provides different narrative styles to address different data narrating approaches.

Additionally, some other web considerations such as browser compatibility and speed of rendering the data are significantly important for a proper online presentation. Statistics shows that 56 percent of adults seek local news on their phone, of which 77 percent are adults between the ages of 18 and 29, representing the highest percentage among all other age segments (Rosenstiel et al., 2011). Therefore, the quality of presentation on smaller screens is a chief factor. Schmitz Weiss $(2013,2015)$ addresses this concept in her article and highlights the importance of creating more news and location-oriented applications for portable devices.

Based on the literature review focusing on cartographic standards and previously emphasized functionalities for a spatial multimedia presentation, we propose the following ten criteria as the most relevant for a good multimedia presentation of spatial journalistic content:

(1) Multimedia capabilities (e.g. audio, video, slideshow, interactive features);

(2) Different narrative structures for digital storytelling;

(3) User-friendly and intuitive GUI that could be used by non-professional users;

(4) Compatibility with different spatial data types;

(5) Spatial analysis tools;

(6) Browser compatibility;

(7) Extensive framework for developers (e.g. API);

(8) Support for mobile devices;

(9) Speed of rendering in browser and server;

(10) License and pricing.

These concluded criteria have no order of importance. The criteria were ranked in a web mapping survey and ultimately influenced selecting the proper platforms for the test story which is described in the chapter 4.

\section{Web mapping survey}

In order to rank these ten criteria, we conducted a webbased survey among 53 participants with journalistic background, gathered from relevant social media groups and mailing lists. Most responses, 38 out of 53, were gathered through the subscription to the mailing list of the "National Institute for Computer Assisted Reporting (NICAR)".
We found that browser compatibility, mobile support and rendering speed are the most important criteria, for creating and publishing a story, while spatial analysis tools seem to be of minor importance among this target group of data journalists (see Figure 1).

The survey participants confirmed the importance of the proposed features, since nine out of the ten features were rated between "very important" to "rather important". Additionally, none of the participants added any further characteristics to the proposed list of criteria. More interestingly, "spatial analysis tools", which is considered a very essential feature in areas such as cartography or geographical science, was placed at the least level of importance from a journalistic point of view.

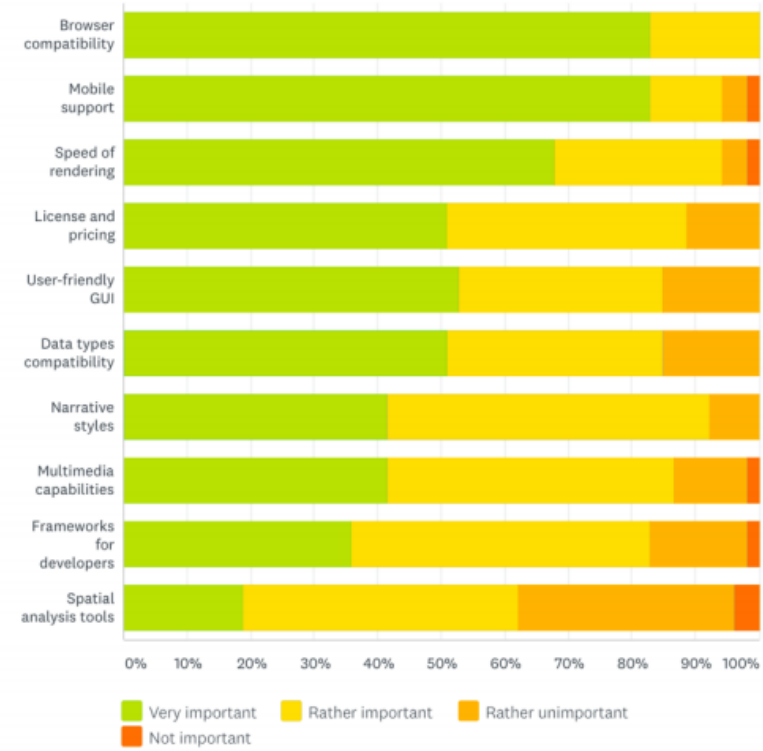

Figure 1. The most important criteria for creating integrated multimedia-web mapping applications.

We also asked the participants which web mapping platforms they use for presenting journalistic content in their daily lives. Although Google Maps was regarded as the most used web mapping platform, it cannot be regarded as a complete platform according to our ten criteria.

The platforms of CARTO, Mapbox and Esri Story Maps were selected as the next alternatives. Additionally, besides these platforms, users named Tableau and Datawrapper quite frequently as their main platform in the comment section. Furthermore, Leaflet, Google Maps API, and D3 were chosen by the participants as the top three most popular web mapping technologies.

The main idea behind this survey was to identify the most suitable web mapping platforms which fulfill the desired considerations for a multimedia presentation. Consequently, the two platforms of CARTO and Esri Story Maps meet the most expected criteria best. Hence, we chose those two platforms for creating our sample story. 


\section{Sample Story}

The two platforms of Esri Story Maps and CARTO were used separately to build a sample story with a number of multimedia elements. The multimedia elements can be categorized as: text, graphics, video, infographics, interactive maps and animation. In this sample story, we wanted to convey how women's social roles have changed during the years and introduce some influential women in history. It consisted of five sections through which the end user can navigate by clicking and scrolling.

Both platforms allow the user to customize the story and add their styling preferences to the provided builders. The authors can publish the results as a web application or embed it via an HTML code to a website.

\subsection{Sample story with Esri Story Maps}

For the first story, Esri Story Maps offer a set of story templates based on common story practices with geographical component. The multimedia elements of the story can be uploaded to these ready-to-use builders either from the computer or through online sources. The maps can be created or accessed through an ArcGIS Online account.
For the implementation of this test example, Story Map Cascade was the most appropriate choice, since it is very well designed to combine narrative texts with all other desired multimedia content.

As the result, Esri Story Maps provided all the necessary tools for creation of web maps as well as the body of the story with all other multimedia elements. The result of the test story with Story Maps Cascade can be accessed via this link: http://arcg.is/2zCV238.

\subsection{Sample story with CARTO}

On the other hand, CARTO has no consistent framework for the whole story. It offers CARTO Engine as a configurable builder for creating different types of interactive web maps with multimedia elements. A custom web design has therefore been created and CARTO builder has only been used for the creation of the web maps within the story.

The result of the story with the custom web design and CARTO can be accessed via this link: http://www.womencan.byethost18.com/Index.html.
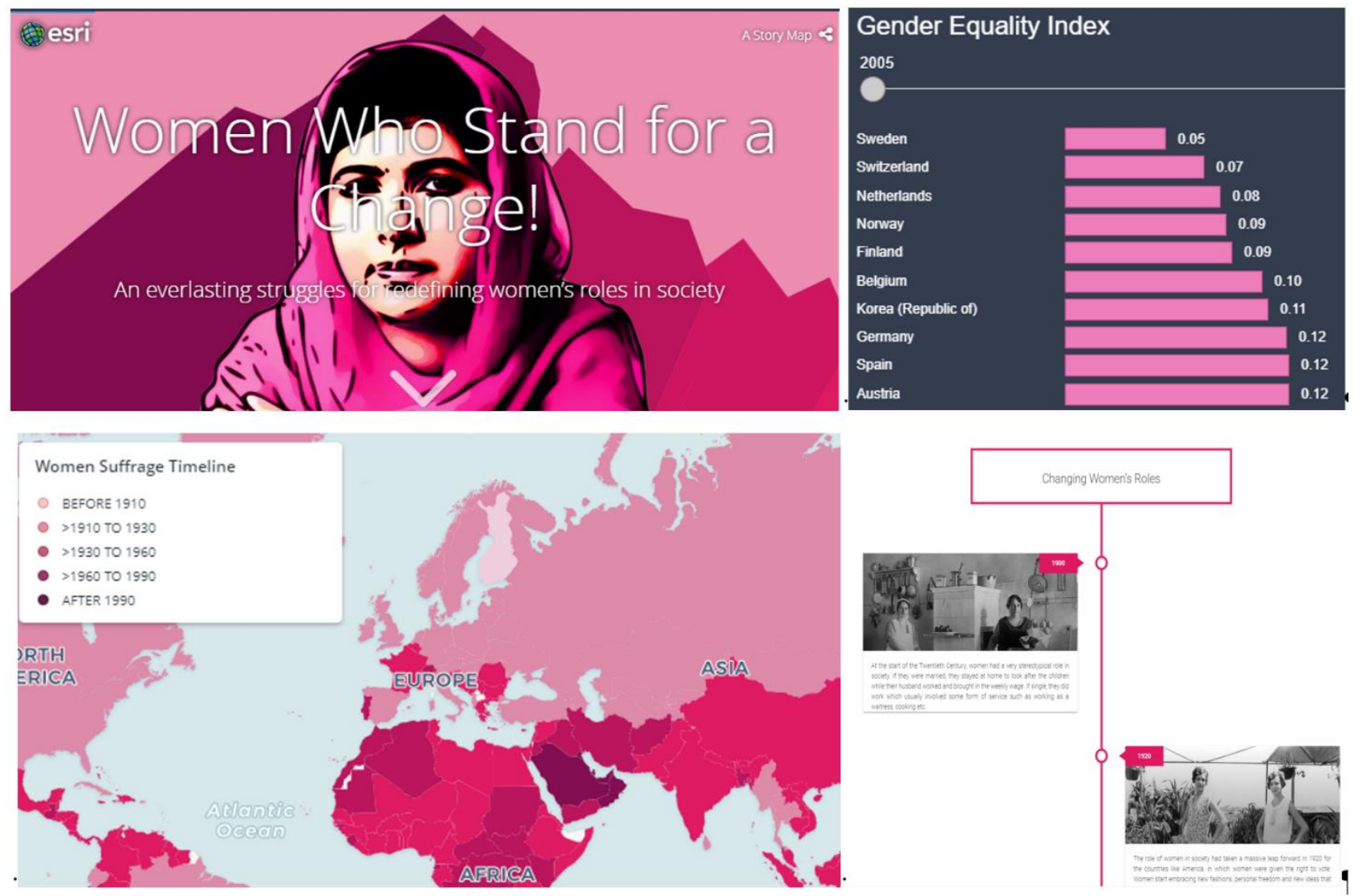

Figure 2. Screenshots from the multimedia presentations of the sample story. 


\section{Results and Discussion}

We found out that Esri Story Maps perform better and provide more services in terms of the overall presentation of multimedia stories with spatial data. However, regarding the creation of web maps of the test story (excluding narrative styles and multimedia capabilities), CARTO showed a better performance. Subsequently, by adding the average ratings of the survey participants to the results of the evaluation, Figure 3 shows in which areas the platforms fulfill the most necessary expectations of the users or not.

For instance, while CARTO addresses the first most necessary features, it fails to fulfill further assistance for creation of a whole multimedia presentations with different narrative styles. On the other hand, Esri Story Maps provide professional analysis tools which are beyond the scope of journalist's usage and need. This could be one of the reasons why this platform is used less among the journalists.

Additionally, it is evident that neither of the selected platforms meet the user's expectations in terms of license and pricing.

In an additionally performed interview with a data journalist, the interviewee found the design of the overall presentation with Esri Story Maps more appealing.
However, he gave more recognition to the web maps that were created by CARTO. One important factor that was highlighted by the journalist was the importance of having the possibility to apply desired customizations to the web maps in order to make it look different from other news broadcasters. This functionality has been addressed in the web interface of CARTO builder, where the users can manipulate data with SQL statements or apply custom styling via CartoCSS in creation of web maps.

Our evaluation showed that while both platforms have their strengths and weaknesses regarding the ten criteria, they are both very useful tools for conveying journalistic stories with a spatial component via the web browser. It can be regarded as a benefit that creating these stories can be significantly facilitated by corresponding web applications. However, to best meet the requirements of journalists in creating such stories, a few modifications like a specific license and pricing list, more customization options and a simpler interface in creating web maps and performing basic special analysis are also suggested to both platforms.

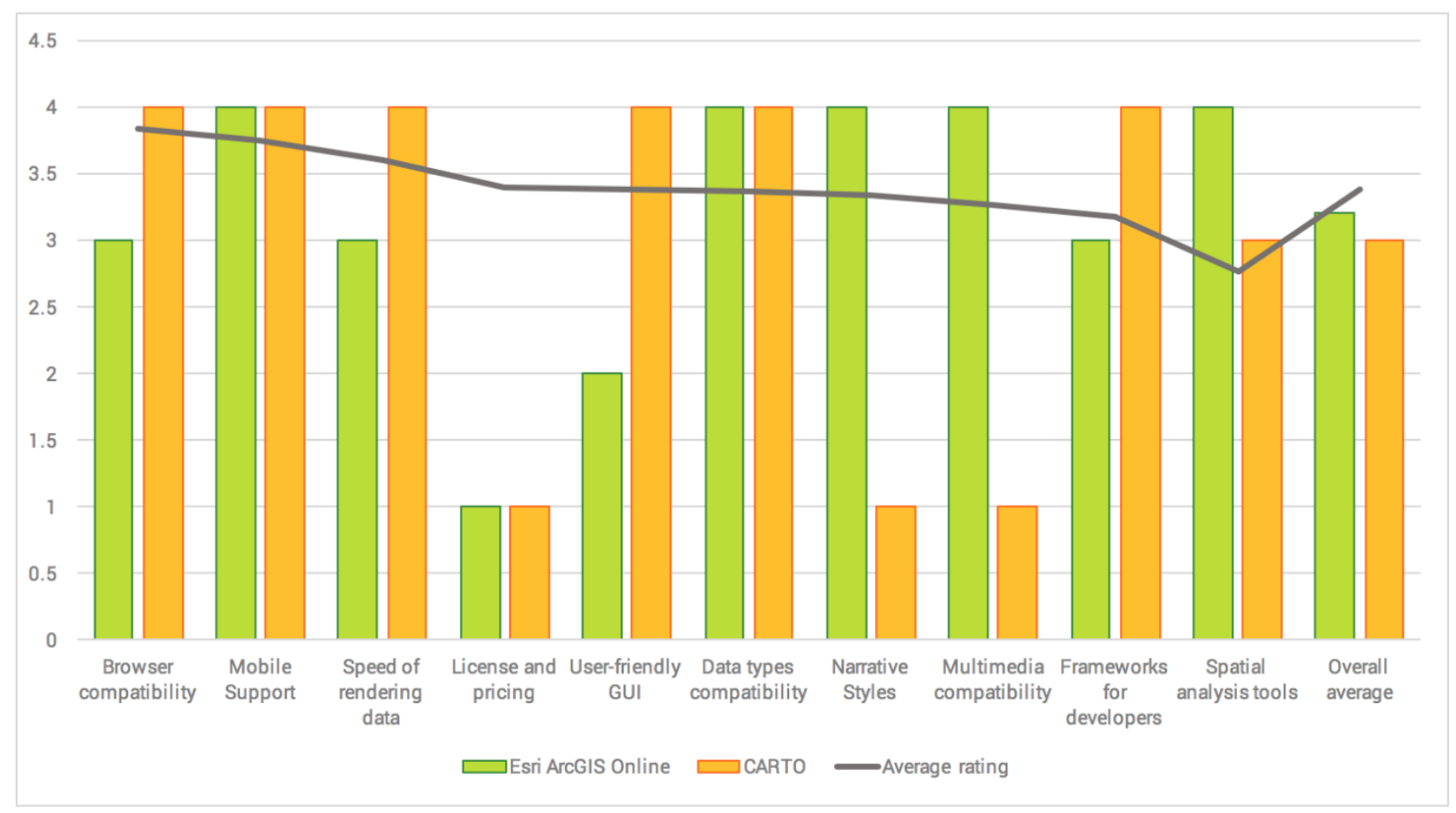

Figure 3: Average ratings for the ten important criteria for the two selected platforms. 


\section{Acknowledgements}

The authors would like to thank all participants of the online survey for their valuable feedback.

A special thank you goes to Esri Germany in Kranzberg for supporting this work which was carried out as Master's thesis research within the international Master of Science program in Cartography www.cartographymaster.eu.

\section{References}

Black, M. and Cartwright, W. (2005). Web cartography \& Web-enabled geographic information systems (GIS)new possibilities, new challenges. In Proceedings of the 22nd International Cartographic Conference.

Churchill, R. R. and Stege, E. H. (2006). From Afghanistan to Iraq in media maps: Journalistic construction of geographic knowledge. Cartographic Perspectives, (54), 55-68.

Feigenbaum, A., Thorsen, E., Weissmann, D. and Demirkol, O. (2016). Visualising data stories together: Reflections on data journalism education from the Bournemouth University Datalabs Project. Journalism Education, 5(2), 59-74.

Jacobson, S. (2012). Transcoding the news: An investigation into multimedia journalism published on nytimes.com 2000-2008. New Media \& Society, 14(5), 867-885.

Rosenstiel, T., Mitchell, A., Rainie, L. and Purcell, K. (2011). Survey: Mobile news \& paying online. The State of the News Media.

Schmitz Weiss, A. (2013). Exploring news apps and location-based services on the smartphone. Journalism \& Mass Communication Quarterly, 90(3), 435-456.

Schmitz Weiss, A. (2015). Place-based knowledge in the twenty-first century: the creation of spatial journalism. Digital Journalism, 3(1), 116-131.

Smiciklas, M. (2012). The power of infographics: Using pictures to communicate and connect with your audiences. Que Publishing. 